\title{
Factors Influencing Patient Satisfaction in Dermatology
}

\author{
Abigail Cline, PhD, MD¹, Tamar Gomolin, BSc², Bijan Safai, MD¹ \\ ${ }^{1}$ Department of Dermatology, New York Medical College School of Medicine, New York, NY \\ ${ }^{2} \mathrm{New}$ York Medical College School of Medicine, Valhalla, NY
}

\section{ABSTRACT}

Background: Patient satisfaction is a proxy for healthcare quality, with physicians evaluated and reimbursed based on patient satisfaction scores. Despite the growing influence of patient satisfaction, factors that impact patient satisfaction in dermatology remain unclear.

Methods: We analyzed 225 responses to an online survey evaluating patient expectations, willingness, and satisfaction regarding dermatology appointments. Patient willingness and satisfaction were measured on a 1-5 Likert scale.

Results: Respondents were most willing to discuss their condition and to be examined with a dermatoscope. Respondents were least willing to wear a patient gown without underwear and to be photographed. Highly satisfying factors included a written treatment plan, provider medication recommendations, and use of gloves during physical exams. Highly dissatisfying factors included waiting 60 minutes, taking off underwear with a patient gown, and being photographed with a cellphone.

Patient willingness and satisfaction differed significantly by gender and age. Male respondents reported less satisfaction than female respondents if a nurse explained the treatment plan. Older respondents were significantly more willing to change into a patient gown, to be photographed, to be examined with a dermatoscope, and to undergo a biopsy than younger respondents. Older and female respondents preferred written plans, wh ile younger and male respondents preferred verbal plans. Younger respondents reported higher satisfaction with an email follow-up compared to older respondents, who preferred a phone call.

Conclusion: These findings may represent relatively easy ways to improve patient satisfaction scores. Further insight into factors affecting patient satisfaction may enhance patient experience and engagement, thereby improving clinical outcomes.

\section{INTRODUCTION}

Enhancing patient experience and engagement may improve clinical outcomes. ${ }^{1}$ Patient satisfaction often serves as a proxy for quality of medicine, with physicians evaluated and reimbursed based on patient satisfaction scores. In the field of dermatology, patient satisfaction plays a very important role since traditional markers of quality, such as mortality and hospital readmission rates, are not reasonable measures of patient care. Patient satisfaction scores are one of the most accessible markers insurance companies 
and prospective patients can use to evaluate dermatologists. The field of dermatology recognizes the importance of patient satisfaction, with the American Board of Dermatology encouraging the collection of patient satisfaction survey data during the Maintenance of Certification process. ${ }^{2}$

Despite the growing research in patients' perceptions of their visits, factors that enhance patient satisfaction remain unclear. While one study found that a patient's perception of the character of the physician directly affects patient satisfaction scores, others have found that environmental factors such as the physician spending a few more minutes with the patient and even the physician sitting down when talking to patients, were relatively easy ways to improve satisfaction scores. ${ }^{3-6}$ However, many of these previous studies were limited by their research methods. One was limited to online patient reviews, which may select towards very positive or negative opinions. ${ }^{4}$ Others were limited to reviews concerning only one dermatology clinic or one hospital system. ${ }^{5,6}$

The goal of this study is evaluate factors related to patient satisfaction with dermatology clinic appointments. Respondents completed a survey assessing patient expectations, willingness, and satisfaction regarding dermatology appointments. To objectively understand factors affecting patient satisfaction, respondents were surveyed independent of their dermatological care using an online survey platform.

\section{METHODS}

After institutional review board approval, an online survey was distributed to individuals older than 18 years-of-age who reported having seen a dermatologist within the past year and having a working knowledge of English. Overall, 225 participants completed the survey via REDcap (Nashville, TN), after recruitment through Amazon's Mechanical Turk (Amazon, Seattle, WA), an online crowdsourcing platform extensively used by psychologists for participant recruitment. ${ }^{7}$ The survey evaluated patient satisfaction using a 1 (no satisfaction) to 5 (a lot of satisfaction) Likert scale. Results were analyzed overall, by age (18-39 years old versus 40 and older), and by gender (female versus male). Statistics included $x^{2}$ tests and Student's t-tests.

\section{RESULTS}

A total of 225 subjects completed the survey (Table 1). Results were analyzed overall, and by demographic groups, including age (18-39 years old versus 40 and older) and gender (female versus male). Overall, satisfaction declined with longer wait times $(p<0.001)$, changing into a patient gown before meeting the dermatologist $(p<0.001)$, and removing underwear $(p<0.001)$ (Table 1). Older patients preferred changing into a gown before meeting the dermatologist, while younger patients preferred the dermatologist to present them with the choice to wear a gown $(p<0.05)$. Older patients also showed a strong preference toward keeping underwear on compared to younger patients $(p<0.05)$. Female patients preferred to be given the choice whether to change into a gown $(p<0.05)$ and to keep underwear on $(p<0.001)$ as compared to male patients.

Satisfaction was higher with use of gloves as compared to bare hands $(p<0.001)$ and with taking pictures using a camera as compared to a cellphone $(p<0.001)$ (Table 1). Older respondents reported higher satisfaction with whole body examination November 2020 Volume 4 Issue 6 
Table 1. Factors Related Patient Satisfaction Before, During, and After the Clinic Visit

\begin{tabular}{|c|c|c|c|c|c|}
\hline & $\begin{array}{l}\text { Overall } \\
(n=225)\end{array}$ & $\begin{array}{l}\text { Age 18-39 } \\
(n=152)\end{array}$ & $\begin{array}{c}\text { Age }>40 \\
(n=73)\end{array}$ & $\begin{array}{l}\text { Female } \\
(n=140)\end{array}$ & $\begin{array}{c}\text { Male } \\
(\mathrm{n}=85)\end{array}$ \\
\hline 10 min wait & $3.0^{*}$ & 3.0 & 3.0 & 3.1 & 3.0 \\
\hline 30 min wait & $1.9^{*}$ & 1.9 & 1.8 & 1.9 & 1.9 \\
\hline 60 min wait & $1.5^{*}$ & 1.5 & 1.3 & 1.5 & 1.5 \\
\hline $\begin{array}{l}\text { Putting on patient gown before meeting the } \\
\text { dermatologist }\end{array}$ & 2.7 & $2.6^{*}$ & $2.9^{*}$ & 2.8 & 2.7 \\
\hline Patient decides whether to put on patient gown & $3.7^{*}$ & 3.7 & 3.7 & $3.8^{*}$ & $3.5^{*}$ \\
\hline Keeping underwear on with patient gown & $3.7^{*}$ & $3.6^{*}$ & $3.9^{*}$ & $3.9^{*}$ & $3.3^{*}$ \\
\hline Removing underwear with patient gown & 2.0 & 2.1 & 1.9 & 2.0 & 2.2 \\
\hline \multicolumn{6}{|l|}{ Satisfaction Factors During the Visit } \\
\hline & Overall & Age 18-39 & Age $>40$ & Female & Male \\
\hline Examining only the area of concern & 3.4 & $3.5^{\star}$ & $3.2^{*}$ & 3.3 & 3.5 \\
\hline Examining the whole body & 3.3 & $3.2^{*}$ & $3.6^{*}$ & 3.4 & 3.2 \\
\hline Touching the skin with bare hands & $2.4^{*}$ & 2.3 & 2.5 & 2.3 & 2.5 \\
\hline Touching the skin with gloves & $4.0^{*}$ & 3.9 & 4.1 & 4.0 & 3.9 \\
\hline Examining with dermatoscope & 3.9 & $3.7^{*}$ & $4.1^{*}$ & 3.9 & 3.9 \\
\hline Taking pictures with cellphone & $2.1^{*}$ & $2.3^{*}$ & $1.9^{*}$ & $2.0^{*}$ & $2.3^{*}$ \\
\hline Taking pictures with camera & $3.3^{*}$ & $3.2^{*}$ & $3.6^{*}$ & 3.4 & 3.3 \\
\hline Performing a skin biopsy & 3.5 & $3.4^{*}$ & $3.8^{*}$ & 3.6 & 3.4 \\
\hline \multicolumn{6}{|l|}{ Satisfaction Factors After the Visit } \\
\hline & Overall & Age 18-39 & Age $>40$ & Female & Male \\
\hline Patient medication recommendations & $3.8^{*}$ & 3.8 & 3.8 & 3.8 & 3.6 \\
\hline Physician medication recommendations & $4.1^{*}$ & $4.0^{*}$ & $4.3^{*}$ & 4.2 & 4.0 \\
\hline Verbal treatment plan & 4.3 & $4.2^{*}$ & $4.5^{*}$ & 4.3 & 4.2 \\
\hline Written treatment plan & 4.3 & $4.2^{*}$ & $4.5^{*}$ & 4.3 & 4.2 \\
\hline Scheduled follow-up & $3.9^{*}$ & $3.8^{*}$ & $4.1^{*}$ & $4.0^{*}$ & $3.8^{*}$ \\
\hline Follow-up as needed & $3.4^{*}$ & $3.5^{*}$ & $3.2^{*}$ & 3.3 & 3.6 \\
\hline Calling the patient after the appointment & 3.9 & 3.8 & 4.0 & 3.9 & 3.8 \\
\hline Emailing the patient after the appointment & 3.9 & 4.0 & 3.8 & 4.0 & 3.8 \\
\hline
\end{tabular}

$(p<0.01)$, use of dermatoscope $(p<0.05)$, and skin biopsies as compared to younger ones $(p<0.05)$. Younger patients reported higher satisfaction having only the area of concern examined $(p<0.05)$ and having pictures taken with a cellphone $(p<0.05)$ as compared to older patients. Male respondents reported higher satisfaction with having pictures taken with a cellphone as compared to female respondents $(p<0.05)$.
Satisfaction was higher when physicians presented definitive medication recommendations as compared to offering patients a range of medication options $(p<0.001)$ and was higher when follow-ups were scheduled in advance as compared to being left to occur as needed $(p<0.001)$ (Table 1). Older respondents preferred verbal $(p<0.05)$ and written $(p<0.01)$ instructions, and scheduled follow-ups $(p<0.05)$ as compared to younger patients. Younger respondents preferred scheduling a

November 2020 Volume 4 Issue 6 
follow-up as needed $(p<0.05)$. Female patients preferred scheduled follow-ups $(p<0.05)$ as compared to male patients.

Overall, the highest satisfying factors included verbal instructions, written instructions, and definitive medication recommendations. Factors rated as the most satisfying differed by age and gender. Older and female patients preferred written instructions, while younger and male respondents preferred verbal instructions. Factors related to dissatisfaction did not differ by age or gender. Factors related to dissatisfaction included long wait times, underwear removal, pictures taken using a cellphone, and use of bare hands during the exam.

\section{DISCUSSION}

These results may elucidate relatively simple ways to improve patient satisfaction scores. Patient satisfaction differed significantly by gender and age, thereby making it more difficult to implement general measures for quality improvement. Limitations of this study include the potential for sampling bias associated with the use of an online survey sample. Nonetheless, this study does overcome common limitations of previous research: the data is not constrained to self-selected online patient reviews, the results are not limited to one dermatological clinic or hospital system, and patients are not being surveyed immediately after appointments, where added pressure can affect responses. ${ }^{5,6,8}$

Taking these findings in a holistic way may shed light on broader principles that clinicians can apply in myriad heretofore undiscussed areas of their practice. It is important to realize that these preferences do not exist as isolated data points, but as markers illustrating broader trends in patient desires. For example, the universal preferences for photography using a dedicated camera rather than a cellphone and for a physician's use of gloves are both consistent with a patient desire for an encounter that is more formal than casual. Similarly, the clear preference patients have against removing underwear is consistent with feelings of physical and psychological vulnerability that is oft discussed by medical ethicists, but perhaps not adequately addressed by practitioners. The cognizant physician may then extrapolate from this to find other things in the examination or treatment routine that may be improved upon.

By contrast, the marked differences relating to how treatment plans are communicated are illustrative of the very real and significant differences in preferred communication styles that other disciplines have long recognized. ${ }^{9}$ It is perhaps of less significance exactly which demographic groups showed which preferences, than it is that such variation in preferences exists, affects satisfaction, and ought to be accommodated.

\section{CONCLUSION}

These findings point to relatively easy ways to improve patient satisfaction scores. Although, the influence of patient experience on overall quality of care is complex and context-dependent, improvements in this realm can be made without jeopardizing other measures of quality. Dermatologists can implement these straightforward changes to enhance patient experience, with the goal of improving clinical outcomes.

Conflict of Interest Disclosures: None

Funding: None 


\section{Corresponding Author:}

Abigail Cline, $\mathrm{PhD}, \mathrm{MD}$

Department of Dermatology

Metropolitan Hospital

1901 First Avenue

New York, NY 10029

Phone: 336-671-9027

Email: aecline25@gmail.com

\section{References:}

1. Camacho F, Balkrishnan R, Khanna V, Khanna $\mathrm{K}$, Feldman SR. How happy are dermatologists' patients. The Dermatologist. 2013;21(4).

2. 2. MOC Requirements https://www.abderm.org/diplomates/fulfilling-mocrequirements/moc-requirements.aspx\#SA.

3. 3. Kincey J, Bradshaw P, Ley P. Patients' satisfaction and reported acceptance of advice in general practice. The Journal of the Royal College of General Practitioners. 1975;25(157):558-566.

4. 4. Uhas AA, Camacho FT, Feldman SR, Balkrishnan R. The Relationship Between Physician Friendliness and Caring, and Patient Satisfaction: Findings from an Internet-Based Survey. The patient. 2008;1(2):91-96.

5. 5. Patel S, Sutton AV, Thorpe J, Tsai KY, Crew $A B$. Patient satisfaction and quality of care: a prospective study at outpatient dermatology clinics. Cutis. 2018;102(1):33-35.

6. 6. De Salins CA, Brenaut E, Misery L, Roguedas-Contios AM. Factors influencing patient satisfaction: assessment in outpatients in dermatology department. Journal of the European Academy of Dermatology and Venereology : JEADV. 2016;30(10):1823-1828.

7. 7. Buhrmester M, Kwang T, Gosling SD. Amazon's Mechanical Turk: A New Source of Inexpensive, Yet High-Quality, Data?

Perspectives on psychological science : a journal of the Association for Psychological Science. 2011;6(1):3-5.

8. 8. Ali ST, Feldman SR. Patient satisfaction in dermatology: a qualitative assessment. Dermatology online journal. 2014;20(2).

9. 9. Pashler H, McDaniel M, Rohrer D, Bjork R. Learning Styles: Concepts and Evidence.

Psychological science in the public interest : a journal of the American Psychological Society. 2008;9(3):105-119 